\title{
Computer-based interactive video systems
}

\author{
LINDA C. PETTY \\ Hampton University, Hampton, Virginia \\ and \\ ELLEN F. ROSEN \\ College of William and Mary, Williamsburg, Virginia
}

\begin{abstract}
Computer-controlled interactive video instruction provides an instructional technique that overcomes many of the shortcomings of using the computer alone as an instructional device. Students who have used interactive video modules have been shown to better retain the material presented and to enjoy the course more than students not using the video modules.
\end{abstract}

The primary sense for the human is vision. The primacy of this modality is reflected in the English language. For example, when we finally come to understand something we say, "I see." Even blind people use this phrase. There are many sayings that refer to the importance of vision, for example, "A picture is worth a thousand words" and "Seeing is believing." Verbal explanation often is not complete until the principle is made visible. In science, all forces are made visual by the instrumentation that we have devised (e.g., the voltmeter and the oscilloscope). Blackboards, transparencies, and slides of maps, diagrams, and graphs are helpful visual teaching aids in any field. One of the boons of the computer is that it allows the presentation of information in visual displays, such as graphs and other graphics. However, visual computer displays are limited and rather crude compared with the "eye feasts" that can be provided using film or videotape. When presenting material on Shakespeare, for instance, the instructor can use films of Shakespeare's dramas to illustrate the point being made. When describing the symptoms of schizophrenia, videotapes of clients displaying typical behaviors can be shown. The microcomputer is now being interfaced with videotape or videodisc players to control the presentation of realistic and engaging visual experiences so that clips can be shown based on the particular program being executed by the computer (see Figure 1). Either videotape of videodisc players can be interfaced with the microcomputer. There are advantages to the use of each medium. Educationally, the videodisc is preferable because it allows random access, whereas tape is, of course, sequential and therefore much slower and limited in the amount of information that can be stored and accessed.

According to Leveridge (1979-1980), "the videodisc represents the most significant innovation in educational technology since the invention of movable type by Gutenburg some 500 years ago" (p. 222). Molnar (1979)

Send reprint requests to Linda C. Petty, Department of Psychology, Hampton University, Hampton, VA 23668.

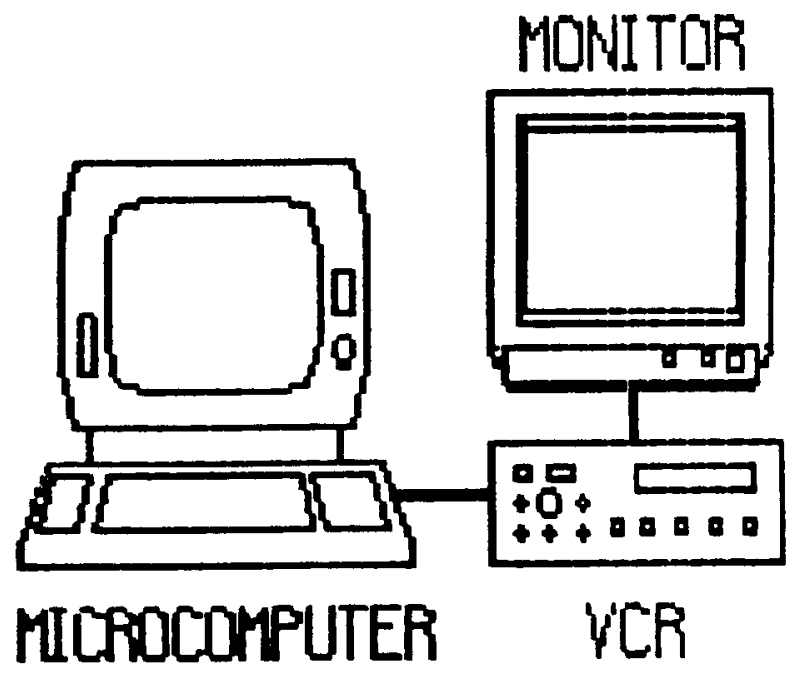

Figure 1. Basic workstation.

describes interactive video as the major way to prevent the current "information boom" from becoming an "information explosion." At the University of Alabama, those students who used interactive video modules in an accounting class not only retained significantly more information, as measured by the final exam, but also enjoyed the course more than did students instructed using traditional materials (Schmidt, 1982). Molnar (1982) found that $30 \mathrm{~min}$ of interactive video instruction was equivalent to $10-15 \mathrm{~h}$ of student studying. Leveridge (1979-1980) found that medical students felt that interactive video experiences were enjoyable and stimulating and that they made good use of student time. IBM ("Videodisks," 1982) discovered that students tend to get bored with traditional computer-assisted instruction (CAI) after about $20 \mathrm{~min}$, whereas interactive video held the students' attention for $54 \mathrm{~min}$. Numerous studies have demonstrated that students learned faster, made higher test scores, and retained more material when interactive video was used in the training process. 
The marriage of these two electronic technologies can potentially increase the appeal and effectiveness of educational materials through the enhancement of active participation of the learner in the educational process. Using the video medium in this fashion also permits the use of high-quality computer-generated graphics. Most highresolution graphics are too slow for effective use in CAI, but when videotape or videodiscs are used, high-powered computer graphics systems can be used to generate the graphics to illustrate the materials and can then be transferred to videotape of videodisc for instantaneous showing.

\section{INTERACTIVITY IN COMPUTERIZED INSTRUCTION}

The level of interactivity required of the learner is significantly increased in interactive video experiences. Interactivity means that the user actively participates in the learning situation and that the user has at least some control of the information presented. These two features require concentration, ensuring that the student pays more attention to the material. Active participation enhances learning. In learner/computer interactions, some response is required that indicates that the learner has mastered or understood some bit of instruction before instruction continues. In the best examples of this type of interaction, the responses of the learner are used to tailor further instruction to the particular needs of this learner. Good measures of the quality of educational software include its ability to process and respond adequately to a broad range of learner input and its capacity to tailor program action to the individual needs and abilities of the learner as revealed through that input. Thus the greater the ability of the program to process natural language and to tailor instruction to the particular characteristics of the learner, the higher marks it gets.

\section{LINEAR FLOW}

The linear model is the least complicated program. Bits of information are printed on the screen of a CRT, and then the message "PRESS RETURN TO CONTINUE" appears at the bottom of the screen. The user can press the return key without even reading the information on the screen. This type of program demands the least involvement of the user. All learners get the same instructional steps in the same sequence. The program designer allows for only one way to master the material in this simple linear approach.

In Figure 2, linear program flow is still evident, but a question is asked after the material to be learned has been presented. If the learner answers correctly, he/she proceeds to the next bit of instruction; if not, he/she loops back and the information is presented again. This model is a review model in that the material is simply repeated and not modified based on the learner's input. Clearly,

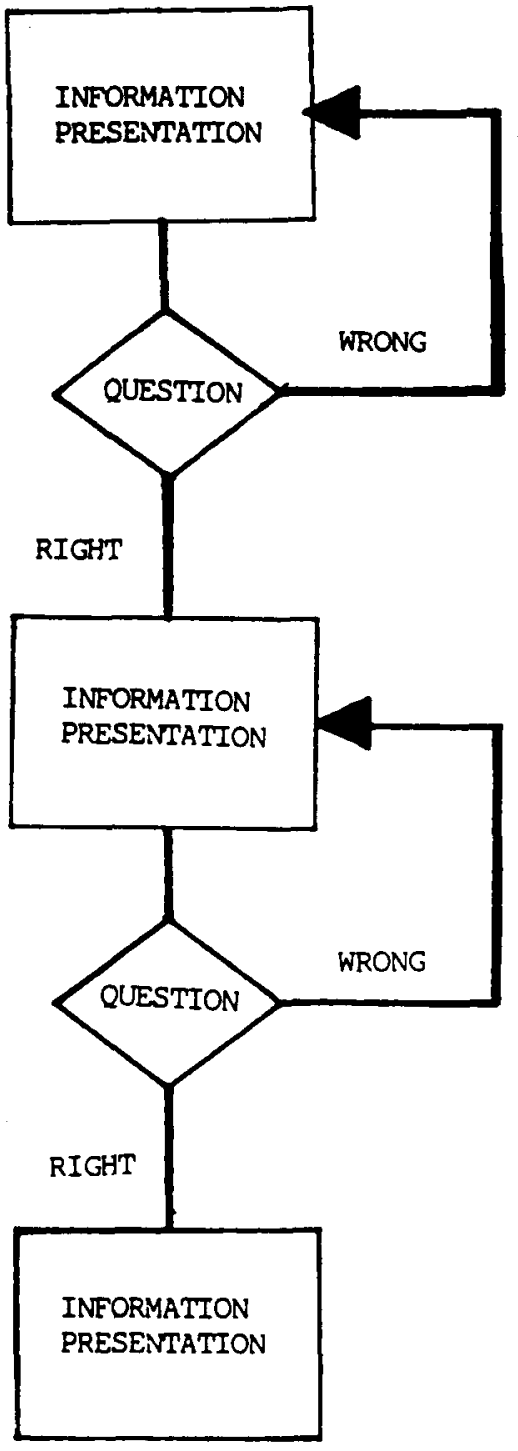

Figure 2. Complex linear model.

this type of program requires greater learner involvement than does the simple linear model.

In a simple branching or remediation model, as shown in Figure 3, a single main path to mastery is still evident, but with questions for the learner to answer after each bit of information is presented. If the answers are correct, the learner continues along the main pathway. If, however, the answers are not correct, the learner is branched, based on the particular mistake that he/she made, to a point in the program where instruction tailored to overcome his/her misconception is presented. Novices in any area tend to make the same errors, so a finite number of branches cover most learners' errors.

\section{NONLINEAR FLOW}

Since single main paths are assumed in the linear models, very little user control is allowed. Some subjects 


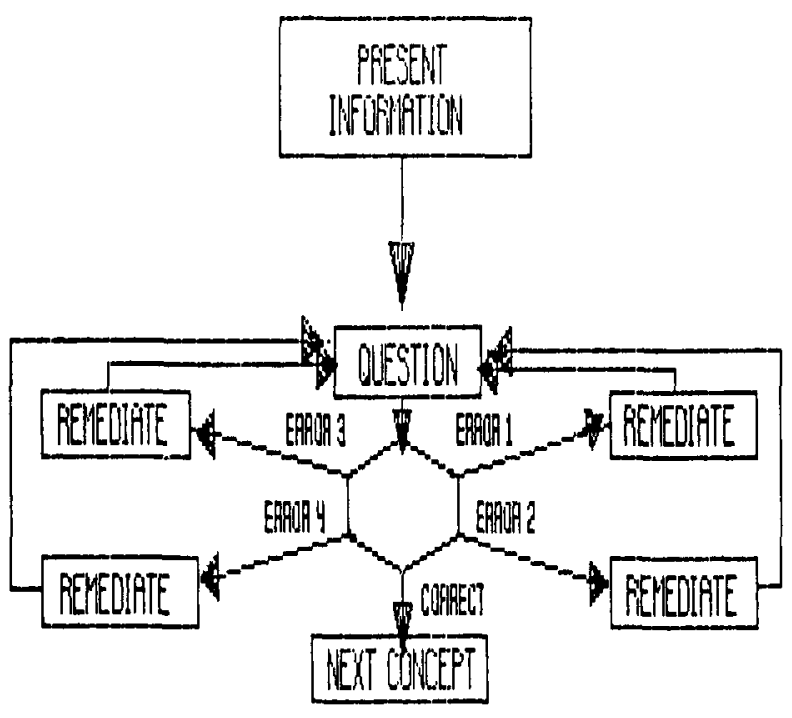

Figure 3. Simple branching or remediation model.

do learn in such a way as to necessitate a linear approach; however, that is not the way we learn most things. Figure 4 presents a model that includes several levels of hierarchical instructional material divided into coherent bits and joined to all the other bits above and below it in such a way that the learner can move in any direction at will. This model allows learner control and does not assume a proper sequence of instruction or a single acceptable instructional outcome. Few educational programs use this model because they are exceptionally complex to write.
The fifth model of learner/computer interaction, the simulation model, is presented in Figure 5. In this scheme, there must be a computerized model of the system to be simulated, whether it is a factory or an experiment, and enough programmed guidance so that the learner reaches the instructional objectives as he/she explores the simulated system. The ideal simulation provides the minimum amount of guidance to achieve successful mastery and the maximum amount of freedom to explore and develop an understanding of the system. Many of the educational computer programs our students rate as the most helpful and the most fun are simulations. A simulation is designed to imitate the functioning of a physical or social system based on some mathematical model of the system. Simulations are of particular benefit when there are resource limitations, such as time or equipment shortages, or when mistakes in real system manipulation place the user or others in peril. Flight simulation is a well-known example. Driver education programs use simulators to place students in potentially dangerous driving situations to test their decision making and reflex actions. Nuclear reactor control room simulators give employees experience in crisis management under possible, but unlikely, circumstances.

Even though the flow of interaction appears to be linear in Figure 6, the learner is in complete control of the system parameters explored and can experience system activities as he/she chooses. In general, these programs allow the user to manipulate variables of the model system and to observe the effect of these changes in the system output. Simulations have the potential of providing an even

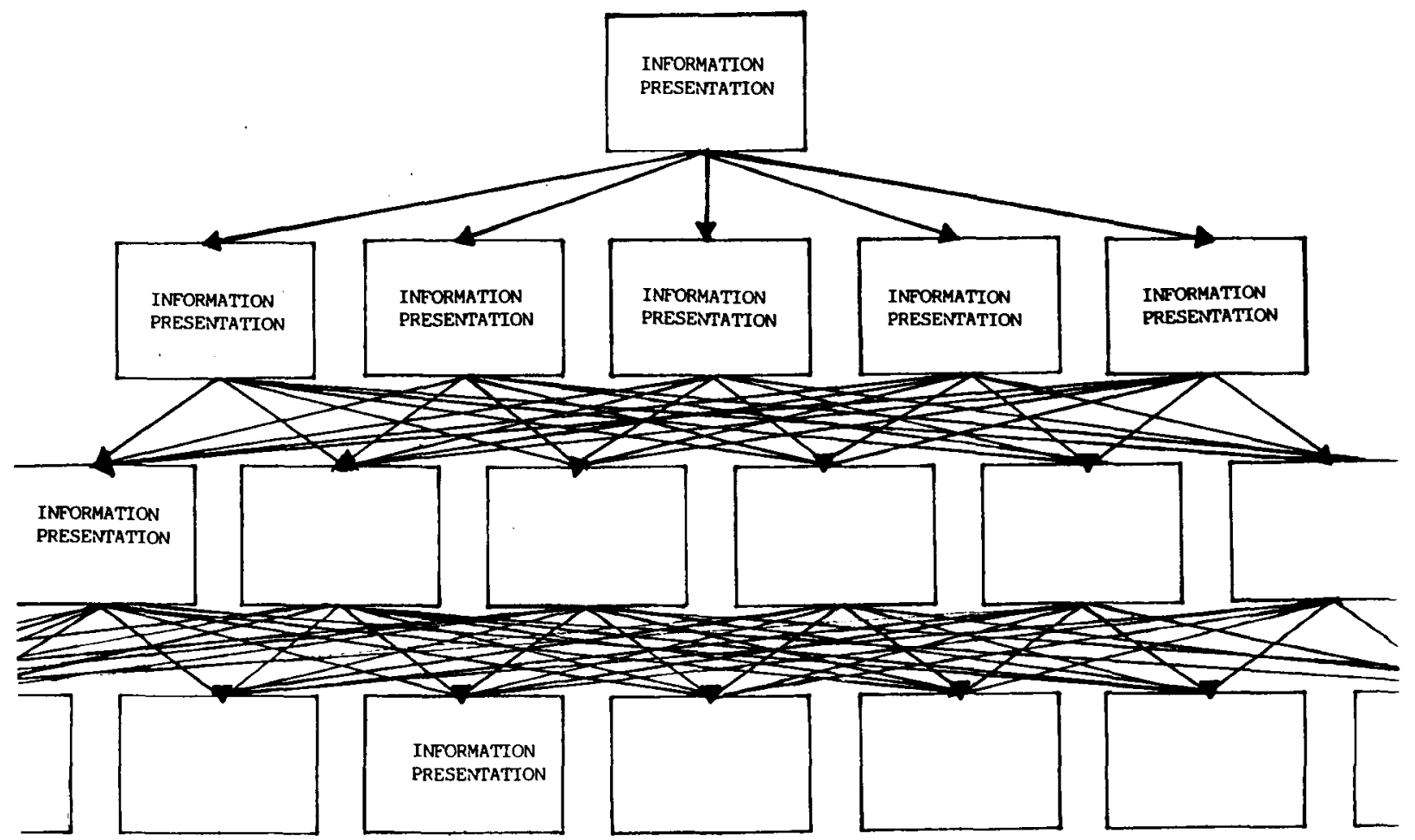

Figure 4. Nonlinear hierarchical model. 


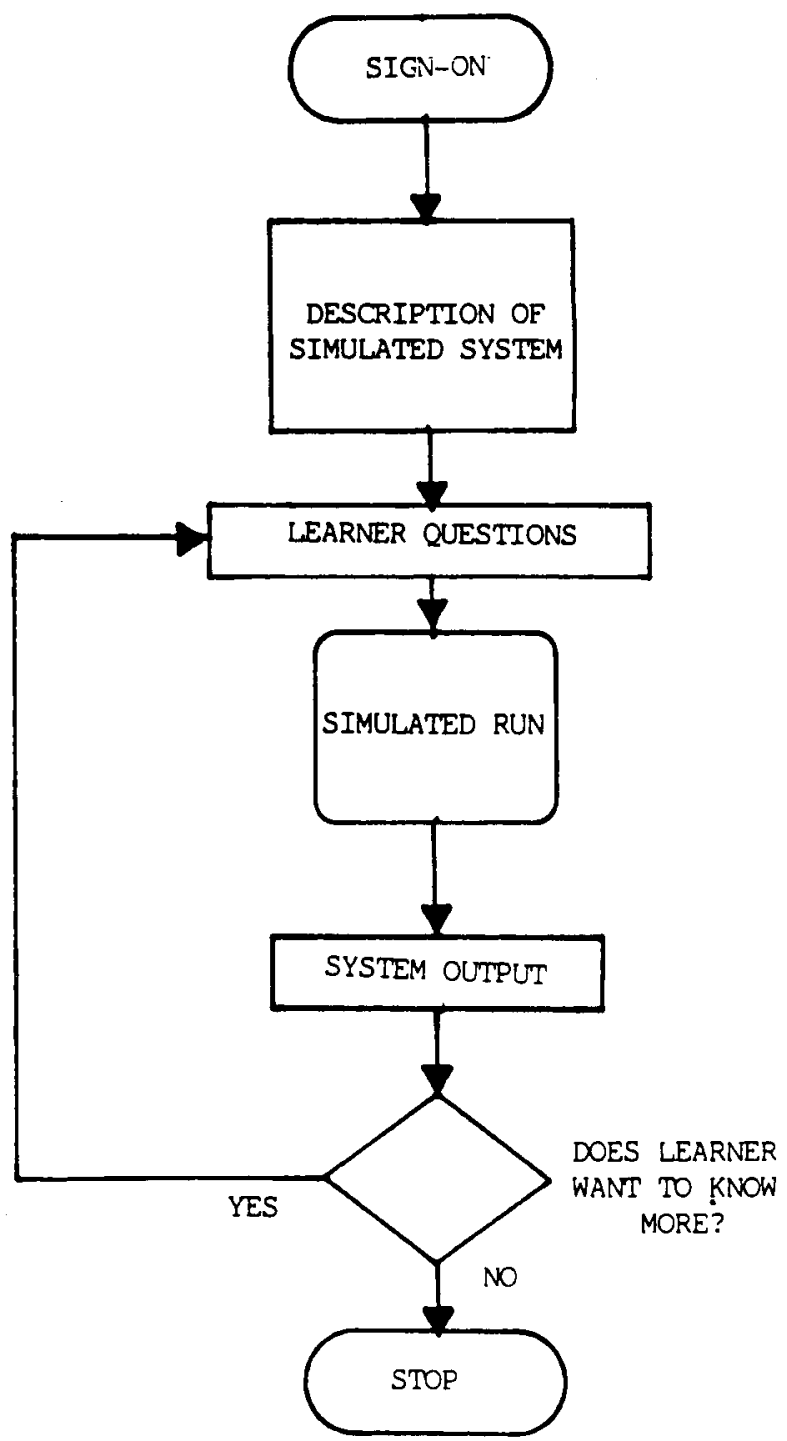

Figure 5. Simple simulation model.

greater mastery of system dynamics than does interaction with the real system, in that many of the important variables in real systems, such as wind speed and temperature, cannot be manipulated at will and can only be studied when the extremes occur naturally. In a computer simulation, various extreme conditions and even rare occurrences can be staged so that the user can learn under those system conditions. In a simulation of the kepone pollution problem in the James River, the turn-over time for bottom sediment can be determined under various wave and weather conditions.

The computer-based tutorial represented in Figure 7 is programmed to preclude misunderstandings by not allowing users to proceed until mastery of each concept has been demonstrated through accurate input from the user. The difficulty level is varied based on user input. Care should be taken to make dialogue as natural as possible and to avoid incorrect grammar and usage in the service of simplicity. This type of software is extremely time- consuming to write, but it is very rewarding when completed because students enjoy these user-friendly programs.

\section{ARTIFICIAL INTELLIGENCE}

Artificial intelligence can be used with the tutorial program to enhance its ability to individualize instruction. However, it is significantly more complex and requires special knowledge of programming languages and concepts. Expert systems are being developed in many fields where problems are solved using a particular body of knowledge and a finite number of relational rules. Work is being conducted to develop an intelligent tutor that can process English input from users and produce conversational dialogue that cannot be distinguished from a hu-

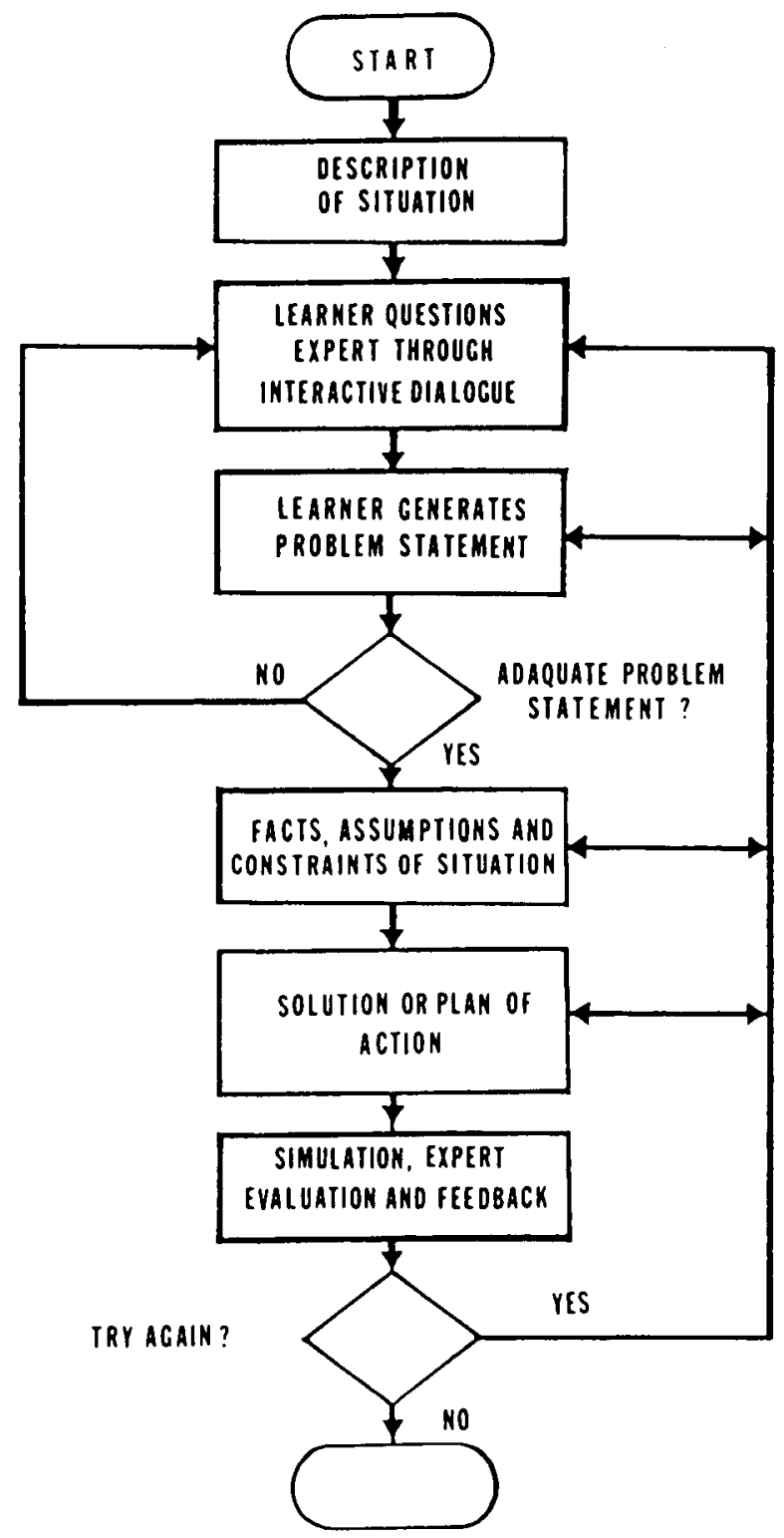

Figure 6. Problem-solving simulation model. 


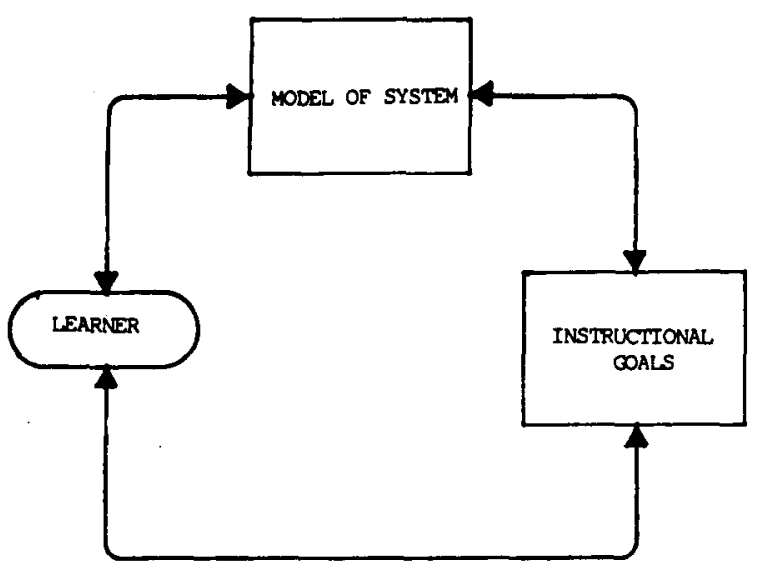

Figure 7. Computer-based tutorial model.

man tutor. This type of educational software has unlimited potential for increasing mastery levels of all users, because the tutor would be programmed to tailor the educational presentation to the cognitive style, the level of intellectual maturity, and the interests and background of each user, and to increase the level of difficulty of the material based on user responses and user characteristics. When these design techniques are used in interactive video experiences, the educational results will surpass those already reported.

Figure 8 includes the major components of such a system. A model of an expert in the field to be mastered must be developed using a complete task analysis of the subject matter to be learned, as well as all relational rules, rules of thumb, and problem-solving strategies necessary. A model of a tutor, including teaching strategies, learner response analysis, rules for generation of tutor responses, and techniques for analysis of learning outcomes, must be programmed.

A model of the learner must also be included. Learner profiles are constructed through the use of a set of decision rules. These rules will produce a profile that is used to individualize the learning experience. Examples of such characteristics are cognitive style (e.g., field independence/dependence, locus of control, impulsiveness/reflectiveness), intellectual development (e.g., concrete/ abstract, Piagetian stages), moral reasoning (e.g., Kohlberg's stages), learner sensory preferences (e.g., dominant sense), and learner habits. Scales are used, with numbers attached to each level of these variables, so that each learner can be described by a finite, but complete, set of numerical values that can be used to tailor actual learning interactions to the students' needs.

The decision rules are constructed by conducting a complete task analysis of the critical skills to be mastered and by defining the desired growth pattern for the individual student. This growth pattern can include changes in such variables as cognitive style, level of intellectual maturity, moral reasoning, learning habits, and problem solving strategies, as well as in the existing level of information in a particular subject area. As the instructional interac- tion proceeds, the system constantly assesses the learner's understanding and mastery of the subject matter. When the learner satisfies the criteria set by the module designers, his/her performance profile is updated to reflect the new level of mastery. As the learner interacts with the system, the profile is modified as the learner masters material, as his/her understanding deepens, and as his/her problem solving strategies become more complex and adequate so that growth can be facilitated. Each module, therefore, constantly encourages learners to move consistently and gradually in the desired direction on all important variables. Finally, overall management of the learning interaction must be determined through the decision rules that contain discourse management and techniques for integration of components. Figure 9 provides a flow chart of such an educational system.

\section{INTERACTIVE VIDEO SYSTEM HARDWARE}

Several turnkey interactive video systems are currently available. Examples include the Sony System and the IVIS from DEC. The cheapest system, however, is an IBM clone with an interface (e.g., BCD, Allen, or Systems Impact at around $\$ 1,200$ each) and a videotape or videodisc player. Special attention must be given to choosing the interface, because each microcomputer requires a different one, as do video players. Industrial level players must be used. Before ordering equipment, call the interface representative to ensure compatibility.

\section{AUTHORING LANGUAGES}

Many authoring languages have been developed that allow control of these interfaces. Those that have been available for sometime for CAI now have extensions for interactive video (e.g., SuperPilot for the Apple and PC Pilot for the IBM; Washington Computer Services, 3028

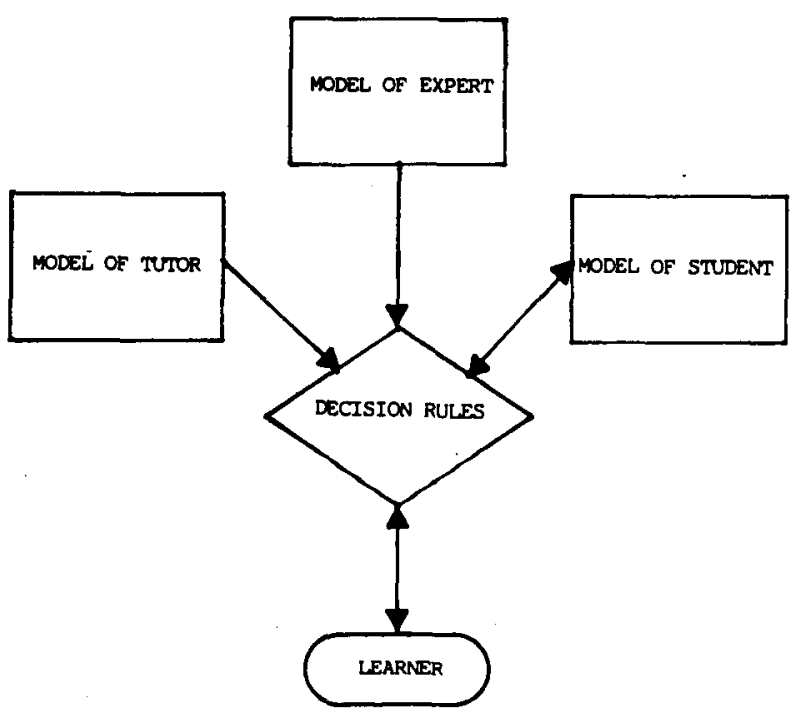

Figure 8. Artificial intelligence model. 


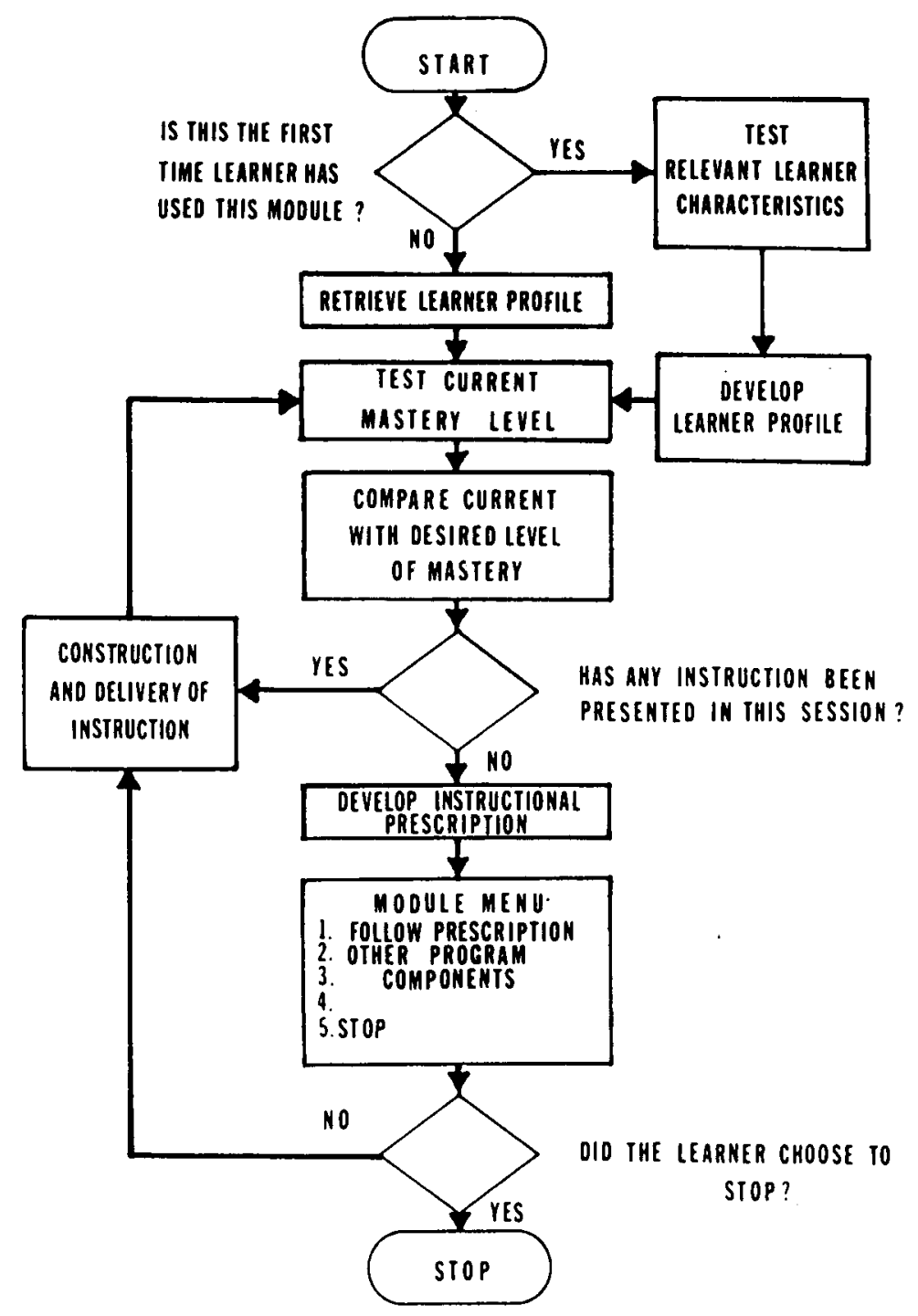

Figure 9. Artificial intelligence model with individualized instruction.

Silvern Ln., Bellingham, WA 98226). Both are excellent development languages that offer many screen and other presentation templates. Developers who generally use BASIC or Pascal may, after trying one of these authoring languages, change because of these screen and other presentation templates.

\section{TAPE VERSUS DISC}

Tape is by far the cheapest development medium. The pressing of a videodisc costs about $\$ 2,000$, which does not include the cost of technical assistance required to shoot the 1-in. master necessary for pressing. Also, this tape must be generated in a very demanding format that is probably beyond the abilities of the media people at most universities. Thus costs can skyrocket. The longterm advantages of videodisc over tape are substantial, however. After the first disc is pressed, copies cost about $\$ 10$ each and last indefinitely. Tapes wear out quickly.
Also, the search time on the random-access disc is about $2 \mathrm{sec}$ for the 55,000 frames. Tape, however, is sequential, and the mechanical devices used to advance and rewind are slow and noisy. The videodisc's excellent image quality and ability to hold still frames without damage to the disc, along with the high density, excellent audio characteristics, and long life of the disc, make discs far more attractive than the tape. Many generic videodiscs are available on a wide range of educational topics and can be used with interactive programs developed for a particular application. Some of these topics include lives and works of major artists, holdings of major museums, animal behavior, architecture, space exploration, photography, Library of Congress holdings, medical diagnosis, and pathology.

At present, use of videotape or an existing disc for a small project makes sense. If, however, you wish to develop a large-scale interactive learning experience to be used by many students in an area where information to 
be mastered has a relatively long life, the cost of generating a videodisc can certainly be justified through amortization over students and time.

\section{INTERACTIVE VIDEO DESIGN AIDS}

A wide range of materials are available to help in getting started on an interactive video project. The basic sources include the following:

DEBLOOIS, M. L. (1982). Videodisc/microcomputer course design. Englewood Cliffs, NJ: Educational Technology Publications.

GAYESKI, D., \& Williams, D. (1985). Interactive media. Englewood Cliffs, NJ: Prentice-Hall.

IUPPA, N. V. (1984). A practical guide to interactive video design. White Plains, NY: Knowledge Industry Publications,
StEINBERG, E. R. (1984). Teaching computers to teach. Hillsdale, NJ: Erlbaum.

\section{REFERENCES}

LEVERIDGE, L. L. (1979-80). Experience in educational design for interactive videodisc and quadrasync presentations. Journal of Educational Technology Systems, 8, 221-230.

Molnar, A. R. (1979). Intelligent videodisc and the learning society. Journal of Computer-Based Instruction, 6, 11-16.

Molnar, A. R. (1982, September). The search for new intellectual technologies. Technological Horizons in Education Journal, 104-112.

SchмiDT, W. D. (1982). Educational technology in college teaching. Educational Technology, 22, 46-48.

Videodiscs, microcomputers from integrated systems. (1982, June 24). Infoworld, 4(24), 16-17. 